\title{
Nonrevisiting Paths on Surfaces
}

\author{
H. Pulapaka and A. Vince \\ Department of Mathematics, University of Florida, \\ Gainesville, FL 32611, USA \\ hpulapak@grits.valdosta.edu
}

\begin{abstract}
The nonrevisiting path conjecture for polytopes, which is equivalent to the Hirsch conjecture, is open. However, for surfaces, the nonrevisiting path conjecture is known to be true for polyhedral maps on the sphere, projective plane, torus, and a Klein bottle. Barnette has provided counterexamples on the orientable surface of genus 8 and nonorientable surface of genus 16 . In this note the question is settled for all the remaining surface except the connected sum of three copies of the projective plane.
\end{abstract}

\section{Introduction}

One of the most well-known open problems in the combinatorial theory of polytopes is the Hirsch conjecture, which gives an upper bound on the diameter of the graph of a polytope. The graph of a polytope $P$ is the 1-skeleton of $P$. More specifically, the Hirsch conjecture states that $\Delta(d, n) \leq n-d$, where $\Delta(d, n)$ is the maximum diameter among the graphs of $d$-dimensional polytopes with $n$ facets. A facet is a $(d-1)$-dimensional face. The Hirsch conjecture was formulated by Hirsch in 1957 and reported by Dantzig in his book Linear Programming and Extensions [5]. The conjecture has implications for the complexity of linear programming algorithms like the simplex method. Since the distance between two points on the graph of a polytope $P$ is a lower bound on the number of iterations of an edge-following algorithm for an LP problem with feasible region $P$, the diameter $\Delta(d, n)$ gives the worst-possible complexity for the best-possible edge-following algorithm. A nice survey on the Hirsch conjecture is the paper by Klee and Kleindschmidt [9].

Equivalent to the Hirsch conjecture is the nonrevisiting path conjecture [8] of Klee and Wolfe. If $p$ is a path in the graph of a polytope, a revisit of $p$ to a face $F$ is a pair of vertices $(x, y)$ such that $p[x, y] \cap F=\{x, y\}$, where $p[x, y]$ is the path along $p$ from $x$ to $y$. In other words, $p$ visits $F$ at $x$, leaves $F$, and subsequently, revisits $F$ at $y$. 
Nonrevisiting Path Conjecture. Any two vertices of a polytope $P$ can be joined by $a$ path that does not revisit any facet of $P$.

The Nonrevisiting Path Conjecture is known to be true for three-dimensional polytopes [1] and is open in higher dimensions. Klee and Walkup [10] showed it to be false, in general, for unbounded polyhedra. Klee [7] has asked about the validity of the Nonrevisiting Path Conjecture for more general complexes. Since the underlying topological space of the boundary complex of a polytope is a sphere, it is natural to ask whether the conjecture is true for cell complexes whose underlying space is a sphere. In this regard, the conjecture is true for 2-spheres, but there is a counterexample due to Mani and Walkup [11] for the 3-sphere.

This note concerns the Nonrevisiting Path Conjecture for polyhedral maps. By a surface $S$ we mean a connected, compact 2-manifold without boundary. These comprise the orientable surfaces $T_{g}$ of genus $g$, which are the connected sums of $g$ tori, and the nonorientable surfaces $U_{h}$, which are the connected sums of $h$ projective planes. Let $G$ be a graph embedded on a surface $S$. The closure of a connected component of $G \backslash S$ is called a face. If the faces are all simply connected and the intersection of any two distinct faces is either a common edge, common vertex, or empty, then $M=(G, S)$ is called a polyhedral map. Two distinct faces that satisfy the condition stated above are said to meet properly. A surface $S$ has the nonrevisiting path property if, for any polyhedral map $M$ on $S$ and any two vertices $x$ and $y$ on $M$, there is a path joining $x$ to $y$ that does not revisit any face. Recent research has been directed toward the following question.

Question. Which surfaces possess the nonrevisiting path property?

The nonrevisiting property holds for the sphere [1], [8], projective plane [2], torus [3], and Klein bottle [6], [12]. However, Barnette [4] has recently provided counterexamples for $T_{8}$ and $U_{16}$. In this note we settle the question for all the remaining surfaces except $U_{3}$, the connected sum of three copies of the projective plane. This may also clarify a misconception [6] that the nonrevisiting path property holds for $T_{2}$, the two-hole torus.

Theorem. The nonrevisiting path property holds for the sphere, torus, projective plane, and Klein bottle; it does not hold for all other surfaces except possibly $U_{3}$.

\section{The Counterexamples}

The proof of the theorem stated in the Introduction requires the construction of counterexamples for all surfaces except the sphere, projective plane, torus, Klein bottle, and $U_{3}$. The first counterexample is a polyhedral map $M$ on $T_{2}$. Figure 1 shows 16 faces. The polyhedral map $M$ is obtained by identifying like labeled edges of these faces. In order to conclude that the result is indeed a polyhedral map, it must be verified that:

(1) The neighborhood of each vertex is homeomorphic to a disk (as opposed to, say, two disks pinched together at that vertex). 

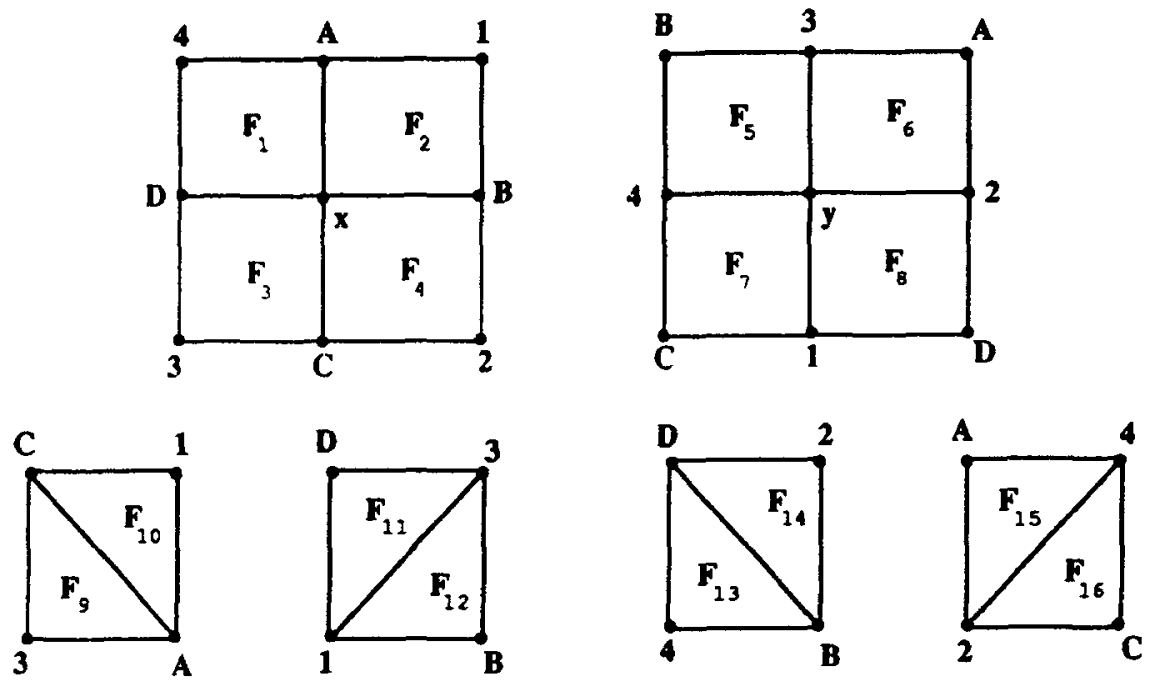

Fig. 1. Counterexample $M$ on surface $T_{2}$.

(2) Pairs of distinct faces meet properly.

(3) The surface is $T_{2}$.

It is easy to check that the boundary cycles of the faces have a coherent orientation, i.e., an orientation such that, for each edge, the directions induced by the two incident faces are opposite. Thus the surface is orientable. Because the surface has 10 vertices, 28 edges, and 16 faces, the Euler characteristic is $\chi=10-28+16=-2$, which implies that the genus is $(2-\chi) / 2=2$. Therefore the surface is $T_{2}$. Conditions (1) and (2) above are easily checked since the example is small.

To show that $M$ does not satisfy the nonrevisiting property, we prove that the vertices labeled $x$ and $y$ in Fig. 1 cannot be joined by a nonrevisiting path. Assume, by way of contradiction, that $p$ is a nonrevisiting path joining $x$ and $y$. Because of the symmetry of $M$ there is no loss of generality in assuming that the vertex adjacent to $x$ along $p$ is the vertex labeled $A$. The path $p$ has now left the faces labeled $F_{3}$ and $F_{4}$ and has visited the face $F_{6}$. Since $p$ is assumed to be nonrevisiting and the vertex $y$ lies on $F_{6}$, the remainder of $p$ must also lie on the face $F_{6}$. There are two ways to get from $A$ to $y$ along $F_{6}$, via vertex 2 or via vertex 3 . If $p$ passes through vertex 2 , then the face $F_{4}$ is revisited by $p$; if $p$ passes through vertex 3 , then the face $F_{3}$ is revisited. Either way leads to a contradiction.

In order to construct a counterexample for the surface $T_{g}, g \geq 3$, let $\bar{M}$ be a polyhedral map on the surface $T_{g-2}$ such that $\bar{M}$ has a triangular face $F_{0}$. The connected sum $M \# \bar{M}$ of $M$ and $\bar{M}$, formed by removing face $F_{0}$ from $\bar{M}$ and face $F_{9}$ of $M$ and identifying the two triangular boundary cycles, is a map on the surface $T_{g}$. Moreover, any two faces of $M \# \bar{M}$ meet properly; otherwise if faces $F$ in $M$ and $F^{\prime}$ in $\bar{M}$ meet improperly, then either $F$ and $F_{9}$ meet improperly on $M$ or $F^{\prime}$ and $F_{0}$ meet improperly on $\bar{M}$. Both are impossible because $M$ and $\bar{M}$ are polyhedral maps. The proof that $x$ and $y$ cannot be 

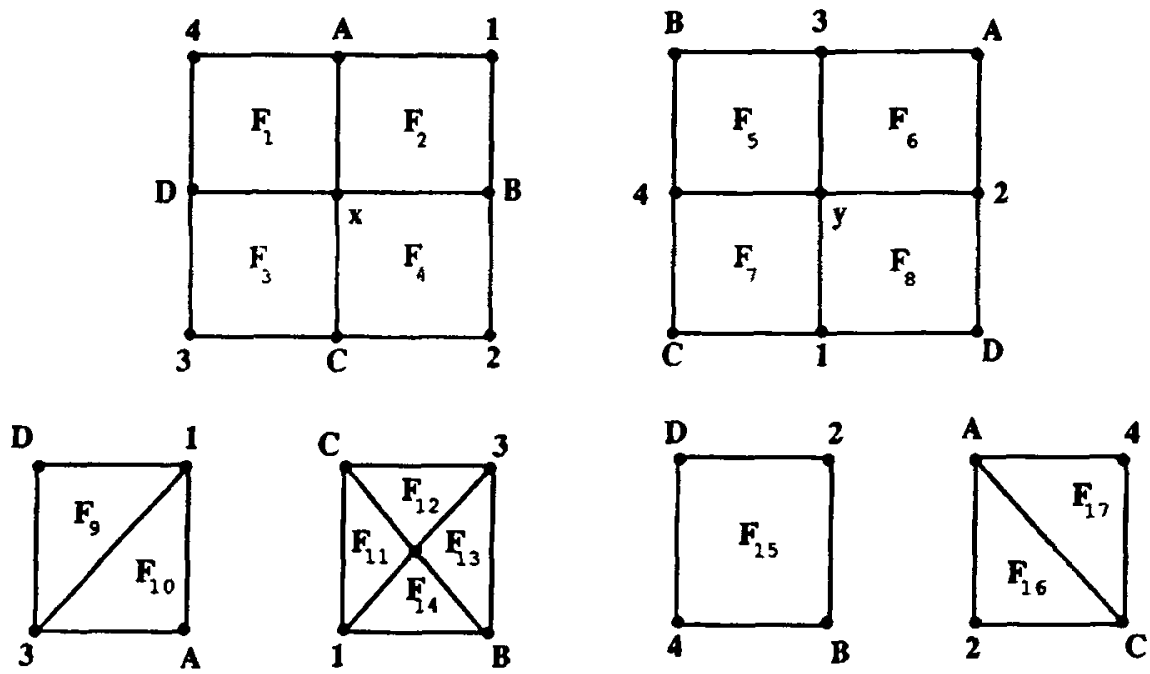

Fig. 2. Counterexample $N$ on surface $U_{4}$.

joined by a nonrevisiting path in $M \# \bar{M}$ is identical to the proof for $M$ alone. Thus the theorem is proved for the orientable surfaces.

For the nonorientable case consider the 17 faces in Fig. 2. As in the orientable case, identify edges with the same labels to obtain a polyhedral map $N$. It is easy to verify that the surface is nonorientable because there is no coherent orientation of the boundary cycles of the faces. The Euler characteristic is $11-30+17=-2$, so the underlying surface is $U_{4}$, the connected sum of four projective planes (or, equivalently, two Klein bottles or a torus and Klein bottle). The proof that $N$ does not satisfy the nonrevisiting path property is identical to the one given for $M$. Counterexamples for the surfaces $U_{h}, h \geq 5$, are also obtained in a manner similar to the orientable case, by taking the connected sum of $N$ and a polyhedral map $\bar{N}$ on $U_{h-4}$.

\section{References}

1. D. W. Barnette, $W_{v}$ paths on 3-polytopes, J. Combin. Theory 7 (1969), 62-70.

2. D. W. Bamette, $W_{v}$ paths in the projective plane, Discrete Math. 62 (1986), 127-131.

3. D. W. Barnette, $W_{v}$ Paths on the torus, Discrete Comput. Geom. 5 (1990), 603-608.

4. D. W. Barnette, A 2-manifold of genus eight without the $W_{v}$-property, Geom. Dedicata 46 (1993), 211-214.

5. G. B. Dantzig, Linear Programming and Extensions, Princeton University Press, Princeton, NJ, 1963.

6. E. Engelhardt, Some Problems on Paths in Graphs, Ph.D. Thesis, University of Washington, Seattle, WA, 1988.

7. V. Klee, Problem 19, Proc. Colloquium on Convexity, Copenhagen, 1965.

8. V. Klee, Paths on polyhedra I, SIAM J. 13 (1965), 946-956; Paths on polyhedra II, Pacific J. Math. 17 (1966), 249-262.

9. V. Klee and P. Kleindschmidt, The d-step conjecture and its relatives, Math. Oper. Res. 12(4) (1987), $718-755$. 
10. V. Klee and D. Walkup, The d-step conjecture for polyhedra of dimension $d<6$, Acta Math. 133 (1967), 595-598.

11. P. Mani and D. Walkup, A 3-sphere counterexample to the $W_{v}$-path conjecture, Math. Oper. Res. 5(4) (1980), 595-598.

12. H. Pulapaka, Nonrevisiting Paths and Cycles on Polyhedral Maps, Ph.D. Thesis, University of Florida, Gainesville, FL, 1995.

Received February 2, 1995. 